\title{
What to Do When the Electromyographer is "Noisy"
}

\author{
Lawrence R. Robinson (10)
}

Keywords: Ground electrode, Electrical noise

doi:10.1017/cjn.2019.56

Can J Neurol Sci. 2019; 46: 468-468

In a recent letter, the ground electrode described her role in noise reduction during electromyographic studies. ${ }^{1}$ This electrode, which prefers to be called E0 rather than ground, serves as a reference point for the E1 (active) and E2 (reference) electrodes. Since voltage is between two points in space, there is no voltage at E1 or E2 alone, and so E0 serves at the reference point. She did not, however, discuss the role that electromyographers play in contributing to some of the noise we see during our studies, or how she can assist us in reducing it.

Most electromyographers have, at times, encountered electrical noise during needle EMG that starts or worsens when the electromyographer touches the needle electrode. The astute electromyographer also notices that it improves when letting go of the electrode. This occurs even via gloved hands (i.e. there is no direct electrical connection). When this happens, the electromyographer should conclude that she/he is personally contributing to the noise level. The purpose of this letter is to discuss why this happens and what to do about it.

As discussed in other sources, ${ }^{2}$ the EMG instrument uses common mode rejection to display the voltage at E1 (referred to E0) minus E2 (also referred to E0). When all goes well, the signal of interest, together with any ambient electrical noise, is recorded at E1-E0, and the ambient electrical noise alone, without appreciable signal of interest, is recorded at E2-E0. If one subtracts the two, then one is left primarily with the signal of interest.

But what if the examiner serves as an antenna, effectively conducting electromagnetic fields from the environment to the needle electrode (E1) that is being held in her/his hand? In that case there is additional noise seen at E1-E0, which is not seen at E2-E0 and that noise is displayed on the EMG instrument.

When this is noted, there are a number of options the electromyographer can consider. One can look for the source of the electrical noise and turn it off or move it further away from the patient. Common sources of noise include: ground loops, nearby electrical equipment (such as lights or electrical beds), electrical main circuits that are not well grounded, broken ground wires, or malfunctioning isolation transformers. One can also ensure there is low impedance at all the recording electrodes and their connections. Most EMG instruments have the ability to measure impedance at the recording electrodes (this is commonly used for evoked potentials). When there is either high impedance or mismatched impedance between E1 and E2, then a better connection should be sought through cleaning the skin, using electrode paste, and making sure the cabling is intact.

But sometimes these measures either are not feasible or are not successful.

This is where our E0 electrode comes in. If E0 can also "see" the noise brought in from the examiner, it can be partially or fully eliminated. How do we do that? We can bring ourselves into connection with E0 by either touching our patient (with skin to skin contact in an area free of body fluids) or we can add an additional ground to ourselves. Many instruments have more than one ground plug and it is easy to put a second ground on the examiner and plug one's self into the ground. This works very effectively.

In conclusion, when noting noise during needle EMG that is better when letting go of the needle electrode, please consider connecting with your E0 electrode. You will thank her for it.

\section{Disclosures}

The author has no conflicts of interest to declare.

\section{Statement of Authorship}

The author is solely responsible for the contents of this article.

\section{REFERENCES}

1. Robinson LR, Christie M, Nandedkar S. A message from the ground electrode. Muscle Nerve. 2016;54(6):1010-11.

2. Dumitru D, Amato AA, Zwarts MJ. Electrodiagnostic medicine, 2nd ed. Philadelphia: Hanley \& Belfus; 2002.

\footnotetext{
From the Health Sciences Centre, University of Toronto, Toronto, Canada.

Received February 6, 2019. Final Revisions Submitted April 5, 2019. Date of ACCEPTANCE APRIL 12, 2019.

Correspondence to: Larry Robinson, Sunnybrook Health Sciences Centre, University of Toronto, St. John's Rehab, S125, 285 Cummer Ave., Toronto, ON M2M 2G1, Canada. Email: Larry.Robinson@Sunnybrook.ca
} 